\title{
The promise of non-invasive prenatal testing needs to be monitored scientifically
}

The name and position of the second author of this Letter was published incorrectly (BMJ 2015;350:h2518, doi:10.1136/bmj. $\mathrm{h} 2518$ ). The second author is Gioacchino Scarano and he is

Unit [not "Scarano Gioacchino, coordinator," as published]. 